GRADIATION\&APPLICATIONS

ISSN 2466-4294 (online) | rad-journal.org

Vol. 3 | Issue 3 | pp. 172-177, 2018

doi: 10.21175/RadJ.2018.03.029

Original research paper

\title{
METAL THIN-FILM DOSIMETRY TECHNOLOGY FOR THE ULTRA-HIGH PARTICLE FLUENCE ENVIRONMENT OF THE FUTURE CIRCULAR COLLIDER AT CERN
}

\author{
Georgi Gorine ${ }^{12^{* *}}$, Giuseppe Pezzullo' , Michael Moll1י, Mar Capeans', \\ Katja Väyrynen³, Mikko Ritala ${ }^{3}$, Didier Bouvet ${ }^{2}$, Federico Ravotti' ${ }^{1}$ Jean-Michel Sallese²
}

${ }^{1}$ European Organization for Nuclear Research (CERN), Geneva, Switzerland

2École Polytechnique Fédérale de Lausanne (EPFL), Lausanne, Switzerland 3 University of Helsinki, Helsinki, Finland

\begin{abstract}
The Future Circular Collider (FCC) design study aims to assess the physics potential and technical feasibility of a new synchrotron accelerator expected to reach an energy level of $100 \mathrm{TeV}$ colliding proton beams circulating in a $100 \mathrm{~km}$ tunnel located in the Geneva area in Switzerland. Inside the FCC detectors, over the 10 years of scheduled operation, unprecedented radiation levels will presumably exceed several tens of MGy with more than $10^{17}$ particles $/ \mathrm{cm}^{2}$. Current dosimetry technologies, such as silicon pin diodes, are not capable of integrating this particle fluence, thus requiring a new type of sensor to be used as dosimeter in future irradiation facilities and, at a later stage, in the FCC accelerator. As a solution for the Ultra High Fluence monitoring, we have focused our research on metal nanolayers. The technology consists of thin film resistive structures deposited on silicon wafers, where sensitivity to displacement damage, measurable in a variation of their electrical properties, can be trimmed by variating geometrical (thickness, $W, L$ ) and physical (material) properties of the nanolayers. The first prototypes of these new dosimeters have been fabricated at EPFL Centre of Micronanotechnology, and specific high-fluence irradiation tests (with gamma, protons, neutrons) have been carried out in several facilities inside and outside CERN. In this paper, after presenting the process flow for the fabrication of these dosimeters, we show the results of annealing tests performed on devices previously irradiated with $23 \mathrm{GeV}$ protons. These measurements suggest the occurrence of an oxidation process that was enhanced by the radiation damage.
\end{abstract}

Key words: Dosimetry, Future Circular Collider, FCC, radiation effects, radiation monitoring

\section{INTRODUCTION}

The European Organization for Nuclear Research (CERN) in Geneva, Switzerland, is presently considering the roadmap of future high energy physics (HEP) experiments and assessing the possible upgrade options that its accelerator complex can offer in the upcoming years. Among different projects, the Future Circular Collider (FCC) is by far the most ambitious of the proposed machines. This $100 \mathrm{~km}$-long synchrotron will supersede today's Large Hadron Collider (LHC) and became the new world's largest and most powerful particle accelerator.

The FCC is going to be able to accelerate particles up to energies of $50 \mathrm{TeV}$ per beam allowing $100 \mathrm{TeV}$ collisions in the detectors at the interaction points (IP) where different experiments will be located. The final version of this machine, the FCC-hh, will allow colliding protons at a peak luminosity of $5 \times 10^{34} \mathrm{~cm}^{-2} \mathrm{~s}^{-1}$, intercepting up to 53000 bunches filled with $2 \times 10^{10}$ particles every $5 \mathrm{~ns}$ [1]. Such great performances come at a cost of generating an extreme radiation environment, several times harsher than in the current LHC. This will pose new challenges for the design and selection of materials, electronic components, and systems to be used in the FCC detectors and in the tunnel. Monte-Carlo simulations were made to estimate the radiation environment cumulated over 10 years of FCC operation. These have shown tens of MGy with more than $10^{17}$ particles $/ \mathrm{cm}^{2}$ in the most exposed layers of the FCC detectors, few $\mathrm{cm}$ from the IP (Figure 1)[2]. These values are more relaxed in the FCC tunnel (KGy, $>10^{15}$ particles $\left./ \mathrm{cm}^{2}\right)[3]$.

In this context, the FCC Radiation Hardness Assurance working group was formed in order to fully understand what radiation environment to expect and to provide qualification protocols, instrumentation and solutions to these radiation issues. Until now at CERN, state-of-the-art dosimeters have been used to monitor the degradation of the electronics and detector components and to perform dosimetry during irradiation tests of these prior to their installation [4-6].

\footnotetext{
* This paper was presented at the Sixth International Conference on Radiation and Applications in Various Fields of Research (RAD 2018), Ohrid, Macedonia, 2018.

** georgi.gorine@cern.ch
} 


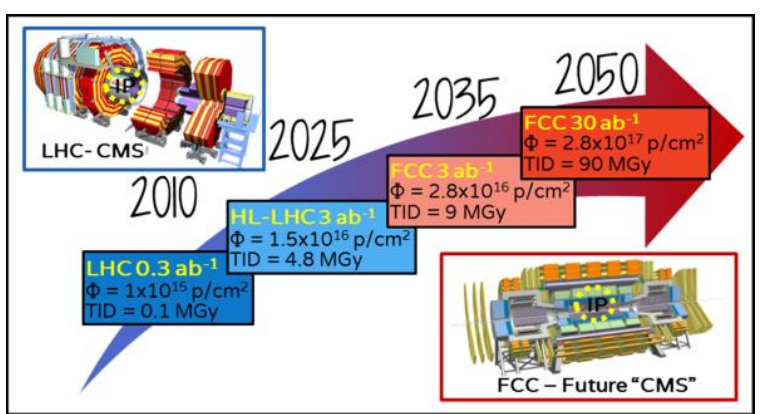

Figure 1. Simulated particle fluence $(\Phi)$ and total ionizing dose (TID), integrated over 10 years, in the inner detectors for different upgrade phases of CERN accelerators.

However, the levels expected in the FCC are several orders of magnitude higher than the ones maximally detectable with any of the currently available dosimetry technology, thus calling for new systems for ultra-high dose and fluence monitoring [7]. Specifically, innovative technologies are being studied in the field of nanometric structures which, thanks to their reduced volumes, are capable of withstanding the harsh radiation environment. In particular, metal thin films are of great interest because they are known to be insensitive to low particle fluence [8] (where current silicon-based devices saturate), and because they have been proven to show several changes of their properties (chemical, mechanical, transmutations, etc.) induced by very high particle fluence [9-11]. An example of such recent developments are, for instance, dosimeters for ultra-high dose monitoring based on optic fibers coated with a thin layer of PMMA and gold, where the dosimetric information is related to shifts in the peak of resonance of such structure [12].

The new technology, presented in this paper, is a dosimeter for ultra-high particle fluence monitoring: the Radiation Dependent Resistors (RDR). In this paper, in Section 2, we firstly outline the easy and inexpensive microfabrication process of the Radiation Dependent Resistors, followed by a description of the irradiation tests that were performed and the temperature measurements. Section 3 details the experimental results and discusses them, whereas Section 4 finally gives the conclusions.

\section{EXPERIMENTAL DETAILS}

All the prototypes of Radiation Dependent Resistors described in this paper were produced in the class-100 cleanroom of the $\mathrm{CMi}$ (Center of Micronanotechnology, [13]) at EPFL (École Polytechnique Fédérale de Lausanne, Switzerland), whereas the electrical characterization, the temperature measurements and most of the irradiation tests were performed at CERN.

\subsection{Microfabrication}

Several wafers were produced with different deposition and/or patterning techniques of the metal layer. At first, we tried different metals such as chromium, aluminium and copper; then, based on the results from the first irradiation tests, we focused our interest on copper only.

As shown in Figure 2, several serpentine shaped devices were designed with different width $\mathrm{W}$, and length $\mathrm{L}$, by varying the number of turns.

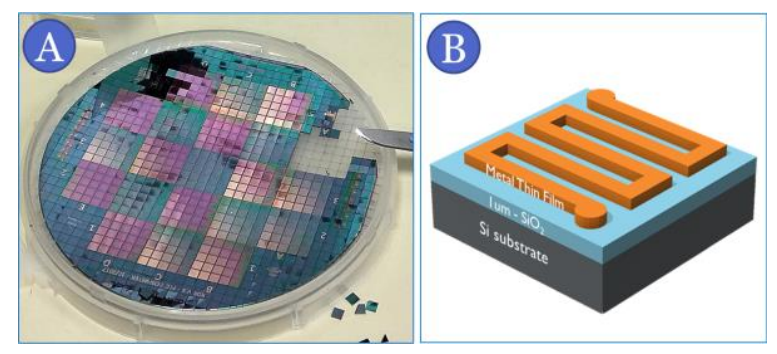

Figure 2. A) Photo of one of the produced wafers with $500 \mathrm{~nm}$ thick copper RDRs with in purple areas passivated with $\mathrm{SiO}_{2}$, B) $3 \mathrm{D}$ model of a single $3 \times 3 \mathrm{~mm}^{2} \mathrm{RDR}$.

Table 1 lists the main fabrication steps followed to produce the Radiation Dependent Resistors. At first, the $100 \mathrm{~mm}$ silicon wafers were exposed to wet oxidation using a Centrotherm Furnace at $1050^{\circ} \mathrm{C}$ for $185 \mathrm{~min}$, which allowed obtaining a $1 \mu \mathrm{m}$-thick insulating $\mathrm{SiO}_{2}$ layer.

The second step was to deposit a thin film of copper, preceded by a Titanium layer needed to enhance the adhesion of $\mathrm{Cu}$ with the underlying $\mathrm{SiO}_{2}$. Two machines were used to obtain a sputtered and an evaporated $\mathrm{Ti} / \mathrm{Cu}$ stack. The Alliance-Concept DP650 sputtering chamber was used at room temperature with a power of $400 \mathrm{~W}$ reaching an average deposition rate for $\mathrm{Ti}$ and $\mathrm{Cu}$ of $3.1 \AA / \mathrm{s}$ and $12.6 \AA / \mathrm{s}$ respectively; the Alliance-Concept EVA 760 evaporator, at a working distance of $450 \mathrm{~mm}$, was used to evaporate $\mathrm{Ti}$ and $\mathrm{Cu}$ at a rate of $5 \AA / \mathrm{s}$. With both machines, copper layers of 50,500 and $1000 \mathrm{~nm}$ were obtained (with Ti adhesion layers of 5,10 and $10 \mathrm{~nm}$, respectively).

The metal deposition was followed by the lithography step. The automatic Süss ACS20o Gen3 coater was used to obtain a uniform $2 \mu \mathrm{m}$-thick layer of Microchemicals AZ9221 positive photoresist, chosen for its high thermal stability during dry etching, for good adhesion and steep sidewalls. The exposure was performed with a Heidelberg Instruments MLA150 direct laser writer providing a dose of $105 \mathrm{~mJ} / \mathrm{cm}^{2}$, followed by the resist development with the Microchemicals AZ40oK developer available in the ACS200.

Table 1. Process flow of a copper Radiation Dependent Resistor microfabrication, with different thin film deposition and etching techniques $(\mathrm{a}, \mathrm{b})$.

\begin{tabular}{|l|l|l|}
\hline \multicolumn{1}{|c|}{ Fabrication Step } & \multicolumn{1}{c|}{ Machine } & \multicolumn{1}{c|}{ Details } \\
\hline 1. Wet Oxidation & Centrotherm Furnace 2.2 & $1000 \mathrm{~nm}$ \\
\hline 2.a Cu Sputtering & Alliance-Concept DP650 & $(10 \mathrm{~nm} \mathrm{Ti}+$ \\
2.b Cu Evaporation & Alliance-Concept EVA 760 & $500 \mathrm{~nm} \mathrm{Cu})$ \\
\hline 3. Photolithography & Süss ACS200 Gen3 & $\mathrm{AZ9221}(2 \mu \mathrm{m})$ \\
& Heidelberg MLA150 & $105 \mathrm{~mJ} / \mathrm{cm}^{2}$ \\
\hline 4.a Dry Etching & Veeco Nexus IBE350 & $34 \mathrm{~nm} / \mathrm{min}$ \\
4.b Wet Etching & Wet bench & $200 \mathrm{~nm} / \mathrm{min}$ \\
\hline 4. $\mathrm{SiO}_{2}$ Passivation & Pfeiffer SPIDER 600 & $300 \mathrm{~nm} \mathrm{SiO}$ \\
\hline 5. Dicing & Disco DAG810 System & $3 \times 3 \mathrm{~mm}^{2} \mathrm{dies}$ \\
\hline
\end{tabular}

To complete the patterning of the RDRs, an ion beam etching was performed with a Veeco Nexus 
IBE350 etcher. The wafers were bombarded with a low power beam at an angle of $10^{\circ}$ achieving an etch rate of $34 \mathrm{~nm} / \mathrm{min}$. Alternatively, a selection of wafers was etched in a solution of Ammonium Persulphate and Sulphuric Acid (6o g/l and $10 \mathrm{ml} / \mathrm{l}$, respectively) achieving an etch rate of $200 \mathrm{~nm} / \mathrm{min}$.

As the last processing step, a selective passivation of some of the devices of the wafers was obtained by sputtering, through a hard mask, of $300 \mathrm{~nm}$ of $\mathrm{SiO}_{2}$ at a deposition rate of $40 \mathrm{~nm} / \mathrm{min}$.

Finally, all the produced wafers were diced using a Disco DAG810 dicing system, resulting in $3 \times 3 \mathrm{~mm}^{2}$ chips (Figure 2.B) ready to be electrically measured using a Süss PM8 probe station equipped with a Keithley 4200A Semiconductor Parameter Analyzer. The selected chips were then wirebonded with a wedgewedge $33 \mu \mathrm{m}$ aluminium wire using a TPT HB10 Bonder on a specifically rad-resistant PCB called FCCRADMON shown in Figure 3.

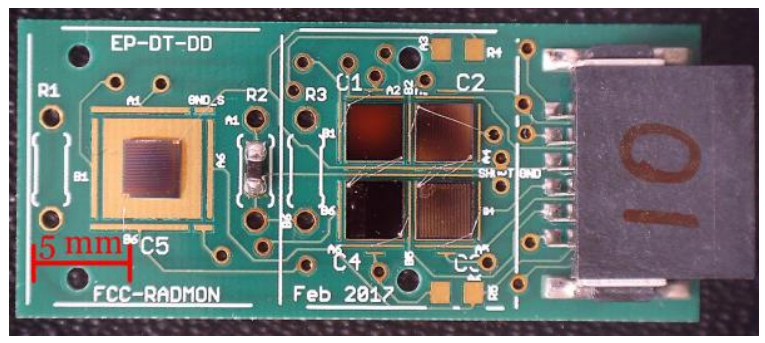

Figure 3. An FCC-RADMON printed circuit board $\left(1.5 \times 3.5 \mathrm{~cm}^{2}\right)$ produced on a radiation resistant Doosan DS-7402 Hal-Free 0.2 mm substrate.

\subsection{Irradiation Tests}

As shown in Table 2, several irradiation experiments were performed (or are still ongoing), to test the RDR sensor performance under conditions similar to the ones in which they will be used. For this purpose, experimental studies were carried out at the TRIGA Nuclear Reactor of the Jožef Stefan Institute (JSI) [14] in Ljubljana, Slovenia, at the Proton Irradiation Facility (IRRAD) [15] at CERN, next to the Linac target in the CERN GBAR experiment [16], as well as next to the luminosity monitors (BRAN) in the Neutral Beam Absorbers (LHC-TAN) of IP1 at LHC [17].

Table 2. List of irradiation experiments already performed and/or ongoing on the RDRs. ( ${ }^{*}$ tests still ongoing)

\begin{tabular}{|c|c|c|}
\hline Test Location & $\begin{array}{c}\text { Radiation } \\
\text { Type }\end{array}$ & Max $\mathbf{\Phi} /$ TID \\
\hline $\begin{array}{c}\text { TRIGA Nuclear } \\
\text { Reactor at JSI }\end{array}$ & Neutrons & $1 \times 10^{18} \mathrm{n}_{\text {tot }} / \mathrm{cm}^{2}$ \\
\hline $\begin{array}{c}\text { IRRAD Proton } \\
\text { Facility at CERN }\end{array}$ & Protons & $1 \times 10^{17} \mathrm{p}^{+} / \mathrm{cm}^{2}$ \\
\hline GBAR at CERN & Gamma rays & $\sim 10 \mathrm{kGy}^{*}$ \\
\hline LHC-TAN at CERN & Mixed field & $\sim 7 \mathrm{MGy}^{*}$ \\
\hline
\end{tabular}

The results from the irradiation campaigns at JSI and IRRAD of 2017, reported in [18], have confirmed our proposed idea of using metal thin films for performing dosimetry of non-ionizing radiation. The test in GBAR at CERN, in a pure ionizing radiation field, is currently ongoing, but is already confirming the ability of the RDR in discriminating the $\Phi$ from TID, being the RDRs unaffected by ionizing radiation (test being performed in CERN-GBAR). Further experiments are currently continuing, as a second run with only the copper samples in JSI and IRRAD, as well as the ongoing test in the LHC-TAN.

\subsection{Temperature Measurements}

Metals are known to be positive temperature coefficient (PTC) materials, meaning that their electrical resistance increases with increasing temperature. While such temperature coefficient $\alpha$ is constant and well-defined for bulk metals, for thin films, the coefficient $\alpha$ is not constant over a wide range of temperatures, and is found to be strongly thickness dependent [19]. For this reason, the prepared FCCRADMON boards were characterized in different ovens, shown in Figure 4, and their temperature characteristics, discussed in Section 3, were extracted before and after irradiation.

The pre-irradiation characterization was performed in a commercial Climats SPIRALE 3 climatic-chamber available at CERN. Such chamber allowed testing samples at different temperatures with a controlled humidity.
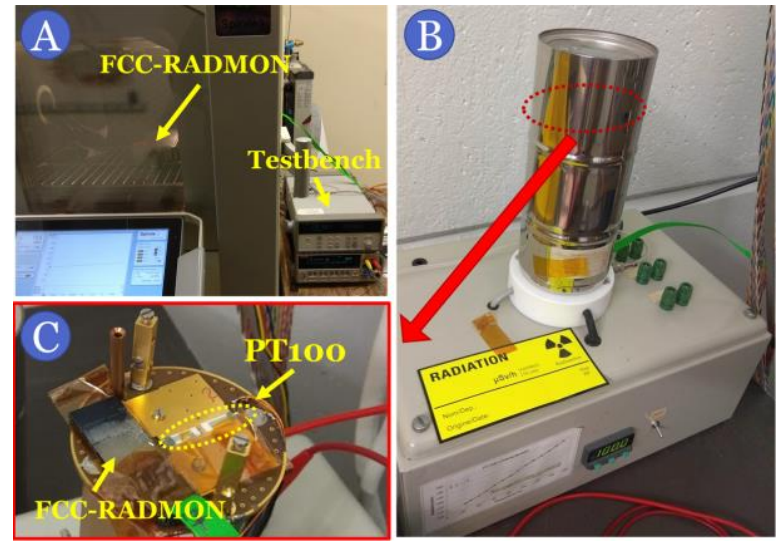

Figure 4. A) Climatic chamber to test samples before irradiation and B) Self-made oven to test after irradiation; C) Detail of the inner part of the cylinder highlighting the PT10o sensors and the FCC-RADMON under test.

The post-irradiation characterization was instead performed in a custom-made oven, shown in Figure 4, which can also be used for radioactive samples. This oven uses a $50 \Omega, 100 \mathrm{~W}$ resistor to uniformly heat a $4 \mathrm{~cm} \varnothing$ support. The power through the resistor is controlled with a power width modulated (PWM) $1 \mathrm{~A}$ current controlled by a proportional-integralderivative (PID) controller. The temperature was monitored using the two PT10o mounted on the sample support, as well as the NTC soldered on the FCC-RADMON PCB.

Measurements were performed over a long period of time (several hours) to reach a stable temperature of $\pm 0.2 \%$ around the setpoint. 


\section{EXPERIMENTAL RESULTS AND DISCUSSION}

Figure 5 shows an extension of the results from the irradiation tests performed between June and December 2017 in IRRAD with $23 \mathrm{GeV}$ protons, on RDRs of copper, aluminium, previously presented in [18].

The experimental data have clearly shown a dependence of RDR resistivity against particle fluence. Such effect can be justified by inspecting the effect of cumulative particle damage: with increasing particle fluence grows the probability of the release of nonionizing energy in the metal lattice, which induces a displacement of an atom from its original position. Such displacement rate, often expressed in literature as displacements per atoms (DPA), introduces defects in the lattice, increasing the electron scattering, thus increasing the resistivity of the metal. This damage resulted to be permanent, without any annealing processes when stored at room temperature. Nevertheless, in order to study the stability of this resistivity increase, we have performed an annealing test which is reported here.

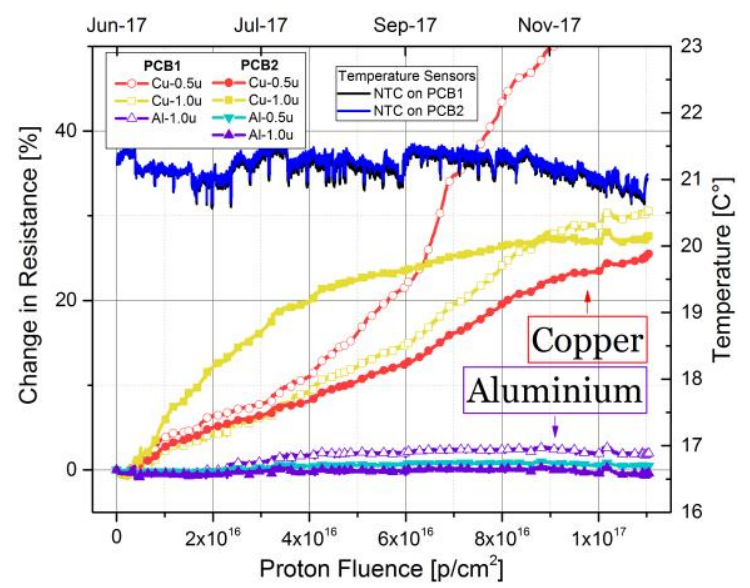

Figure 5. Resistance variation from the initial value in \% (left $\mathrm{y}$-axis) vs the integrated particle fluence. On the right y-axis: the measured temperature during the irradiation test, considerable constant around $21^{\circ} \mathrm{C}$. Experimental data from the RDR irradiation test performed in 2017 with $23 \mathrm{GeV}$ protons in IRRAD, CERN.

\subsection{Temperature Test Results}

Using the experimental equipment described in Section 2.3 and shown in Figure 4, samples were annealed before and after irradiation. The measurements before irradiation (not presented in this paper) were performed with 10 hours annealing in air, ramping at constant rate from $20^{\circ} \mathrm{C}$ to $60^{\circ} \mathrm{C}$ and from $20^{\circ} \mathrm{C}$ to $80^{\circ} \mathrm{C}$. After these measurements, temperature coefficients were extracted for each of the sample. Moreover, none of the 30 different RDRs tested have shown any measurable permanent increase or decrease of resistivity, thus indicating an overall good stability and uniformity of the sputtered metal films.

Concerning the annealing measurements on RDRs irradiated in IRRAD, only the PCB2, shown in Figure 5, has been tested, since the $\mathrm{Cu}-O .5 \mathrm{u}$ sample of PCB1 has

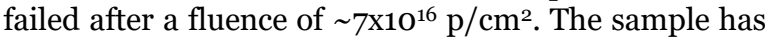
been annealed for about 150 hours, as shown in Figure
6. Such test was performed 5 months after the end of irradiation, once the activation of the sample was reasonably low. During this time, the PCB was stored at $\mathrm{CERN}$ in a radioactive storage at ambient temperature.

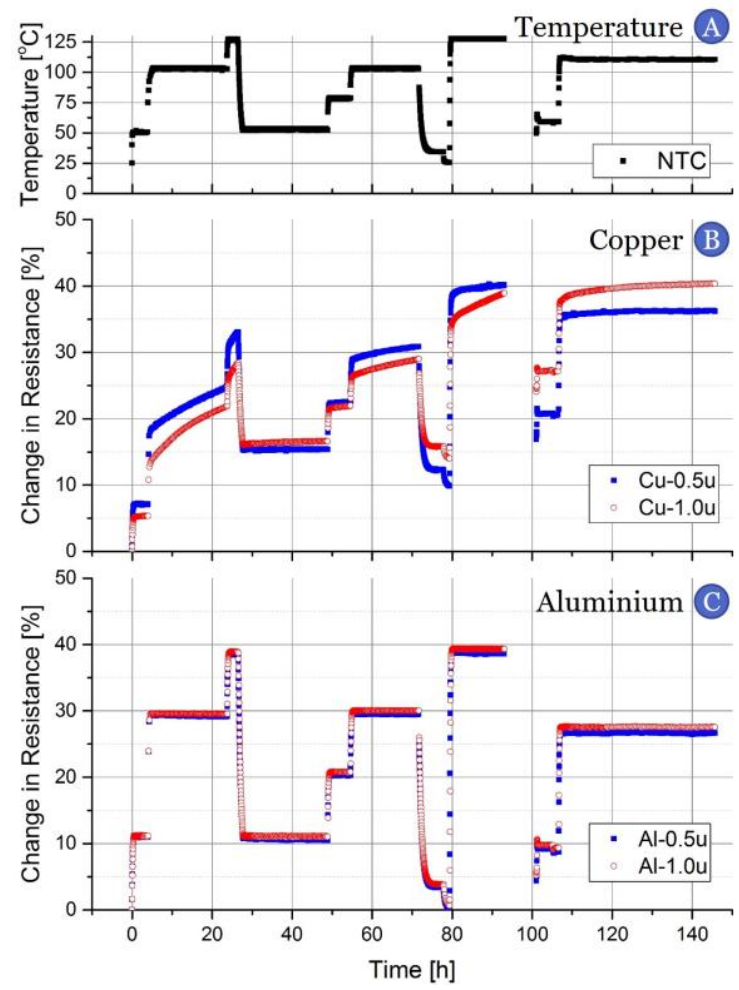

Figure 6. Change in resistance of copper (B) and aluminium (C) vs time at varying temperature (A). Annealing performed on the PCB2 previously irradiated in IRRAD during 2017 run.

As shown in Figure 6, after observing no apparent variation of resistivity for 4 hours at $50^{\circ} \mathrm{C}$, the temperature in the oven has been increased to $100^{\circ} \mathrm{C}$ for 20 hours. During this time, while the aluminium samples (Figure 6.C) have not shown any change in resistance at constant temperature, the copper samples have shown a positive increase (Figure 6.B). This behavior was even more visible when increasing the temperature to $125^{\circ} \mathrm{C}$ for the next 4 hours, at which the slope of change of resistance for copper almost doubled. By further decreasing and increasing the temperature, following the temperature curve in Figure 6.A, the behavior of the aluminium samples remained the same, whereas the copper ones, after more than 100 cumulative hours at high temperature, have shown a saturation of such increasing effect.

An explanation of what has been recorded during this post-irradiation annealing test is an oxidation process of the copper film together with a diffusion of the oxygen-rich copper oxide layer inside the bulk. Normally, metal films create a native oxide layer after exposure to air. In both aluminium and copper, such a layer measures few $\mathrm{nm}$ and, after its formation, it drastically reduces further oxidation at room temperature [20]. While, in case of aluminium, this native oxide layer is shown to be effectively protecting the metal at a wide range of temperatures, whereas in case of copper, at temperatures $\left(200^{\circ} \mathrm{C}-300^{\circ} \mathrm{C}\right)$ the 
oxygen-rich native $\mathrm{CuO}$ oxide layer is shown to penetrate the $\mathrm{Cu}$ layer, creating an enlarging $\mathrm{Cu}_{2} \mathrm{O}$ [21, 22]. This oxidation rate is demonstrated to be dependent on temperature and $\mathrm{Cu}_{2} \mathrm{O}$ grain size, thus on the amount of free diffusional paths available for the oxygen to further oxidize the underlying copper. Such documented effect may have been enhanced by the radiation damage of the film, where displacement damage in the native oxide resulted in an increase in the number of these diffusional paths, resulting in a visible and measurable increase of resistivity of the film even at low temperatures as around $100^{\circ} \mathrm{C}$. Another possible explanation for the resistivity increase is the presence of agglomeration processes induced by temperature and radiation, causing discontinuity in the film. This will be checked upon morphological analysis with a scanning electron microscope (SEM) of the samples before and after irradiation.

\section{CONCLUSIONS}

In this paper, we presented the microfabrication steps, the irradiation tests, and temperature characterization of a novel dosimetry technology for monitoring very high particle fluence. Such device, called the Radiation Dependent Resistor (RDR), has shown an increase of resistance with increasing particle fluence and, differently from silicon based p-i-n diodes commonly used for fluence dosimetry, the RDR has shown no saturation or breakdown until a tested extreme fluence of $10^{18}$ neutrons $/ \mathrm{cm}^{2}$ and $10^{17}$ protons $/ \mathrm{cm}^{2}$. Such behavior confirms the possibility to relate particle fluence to an increase of resistivity of metal thin films.

Annealing tests were performed before and after irradiation. While, before irradiation, no effect was recorded on metals resistivity under prolonged annealing at $60^{\circ} \mathrm{C}$ and $80^{\circ} \mathrm{C}$, after irradiation, copper has shown a continuous and permanent increase of its resistance which started saturating only after 100 hours at $100^{\circ} \mathrm{C}$. Such behavior could be explained by an increase of diffusional paths available to the oxygen for oxidizing the copper bulk samples after irradiation, thus increasing the sites where oxygen can oxidize copper. Such behavior can also be explained by temperature-induced film agglomeration.

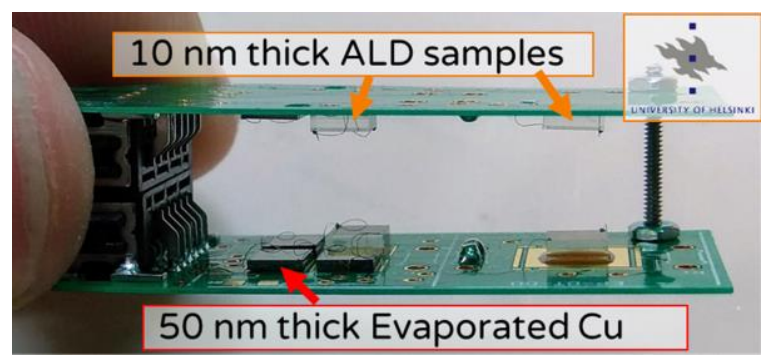

Figure 7. New batch of PCB currently undergoing irradiation tests. New samples of copper RDR were produced, along with ultra-thin ALD samples produced by the University of Helsinki.

Further studies on the effects of particle fluence on copper thin films is ongoing by producing a larger set of different devices, as shown in Figure 7, fabricated with different techniques (e.g. evaporated $\mathrm{Cu}$ instead of sputtered), with different thicknesses (10 $\mathrm{nm}$ to $1000 \mathrm{~nm}$ ), and by passivating some of the devices to exclude oxidation processes during irradiation. Special attention will be given to samples prepared with the atomic layer deposition (ALD) technique, where extremely thin continuous copper layers can be obtained starting from $8 \mathrm{~nm}$ [23].

Finally, an annealing test will be performed in different atmospheres (e.g. helium) to assess the impact of the oxygen concentration on the increase of the oxide layer on the copper thin film, followed by an extensive morphological analysis such as scanning electron microscope (SEM) and atomic force microscopy (AFM) imaging as well as X-ray diffraction (XRD) and X-ray photoelectron spectrum (XPS) techniques, of copper samples before and after irradiation.

Acknowledgements: The authors would like to thank L. Snoj, I. Mandic, A. Jazbec, and S. Rupnik from JSI, Ljubljana, Slovenia, for their help with the neutron irradiation. They would also like to thank all the staff of the CMi cleanroom for their valuable help. This work was supported in part by CERN Future Circular Collider, Special Technologies, Radiation Hardness Assurance, Work Package 11, and in part by the European Union's Horizon 2020 Research and Innovation Programme through the H2O2O Project AIDA-202O Transnational Access under Grant 654168 .

\section{REFERENCES}

1. F. Zimmermann, "High-energy physics strategies and future large-scale projects," Nucl. Instr. Methods Phys. Res. B, vol. 355, pp. 4-10, Jul. 2015. DOI: 10.1016/j.nimb.2015.03.090

2. M. I. Besana, F. Cerutti, A. Ferrari, W. Riegler, V. Vlachoudis, "Evaluation of the radiation field in the future circular collider detector," Phys. Rev. Accel. Beams, vol. 19, no. 11, 111004, 2016. DOI: 10.1103/PhysRevAccelBeams.19.111004

3. A. Infantino, R. Alía, M. Besana, M. Brugger, F. Cerutti, "Preliminary design of CERN Future Circular Collider tunnel: first evaluation of the radiation environment in critical areas for electronics," in 13th International Conference on Radiation Shielding (ICRS-13), Paris, France, 2016. DOI: 10.1051/epjconf/201715303004

4. G. Spiezia et al., "The LHC radiation monitoring system - RadMon," in Proc. 10 $0^{\text {th }}$ Int. Conf. Large Scale Applications and Radiation Hardness of Semiconductor Detectors (RD11), Florence, Italy, 2011. Retrieved from: https://pos.sissa.it/143/o24/pdf; Retrieved on: Mar. 20, 2018

5. F. Ravotti et al., "Conception of an integrated sensor for the radiation monitoring of the CMS experiment at the large hadron collider," IEEE Trans. Nucl. Sci., vol. 51, no. 6 , pp. $3642-3648$, Dec. 2004 DOI: 10.1109/TNS.2004.839265

6. F. Ravotti, "Development and Characterisation of Radiation Monitoring Sensors for the High Energy Physics Experiments of the CERN LHC Accelerator," Ph.D. dissertation, Universite Monpellier II, 2006. Retrieved from: http://cds.cern.ch/record/1014776; Retrieved on: Mar. 20, 2018 
7. B. Camanzi, A. Holmes-Siedle, "The race for new radiation monitors," Nat. Mater., vol. 7, pp. $343-345$, May 2008.

DOI: $10.1038 /$ nmat2159

PMid: 18432200

8. H. Shulman, W. S. Ginell, NASA Space Vehicle Design Criteria- (Structures): Nuclear and space radiation effects on materials, NASA, Washington (DC), USA, 1970.

Retrieved from:

https://ntrs.nasa.gov/archive/nasa/casi.ntrs.nasa.gov/ 19710015558.pdf;

Retrieved on: Mar. 20, 2018

9. J. W. Martin, "The electrical resistivity of some lattice defects in FCC metals observed in radiation damage experiments," J. Phys. F: Met. Phys., vol. 2, no. 5, Sep. 1972.

DOI: $10.1088 / 0305-4608 / 2 / 5 / 008$

10. S. J. Zinkle, "Electrical resistivity of small dislocation loops in irradiated copper," J. Phys. F: Met. Phys., vol. 18, no. 3, 377, Mar. 1988 .

DOI: $10.1088 / 0305-4608 / 18 / 3 / 009$

11. R. L. Chaplin, R. R. Coltman, "Defects and transmutations in reactor-irradiated copper," J. Nucl. Mater. vol. 108-109, pp. 175 - 182, Jul-Aug. 1982. DOI: $10.1016 / 0022-3115(82) 90485-8$

12. F. Fienga et al., "Lab-on-Fiber as dosimeter for the ultra high dose scenario," in Proc. IEEE Nuclear Science Symposium and Medical Imaging Conference (2018 IEEE NSS/MIC), Sydney, Australia, 2018.

13. Center of MicroNanoTechnology official webpage, École polytechnique fédérale de Lausanne, Lausanne, Switzerland, 2018.

Retrieved from: https://cmi.epfl.ch;

Retrieved on: Apr. 13, 2018

14. G. Žerovnik, "Validation of the neutron and gamma fields in the JSI TRIGA reactor using in-core fission and ionization chambers," Appl. Radiat. Isot., vol. 96, pp. $27-35$, Feb. 2015.

DOI: $10.1016 /$ j.apradiso.2014.10.026

PMid: 25479432
15. B. Gkotse et al., "A new high-intensity proton irradiation facility at the CERN PS east area," in Proc. Int. Conf. Technology and Instrumentation in Particle Physics 2014 (AIDA-CONF-2014-O19), Amsterdam, Netherlands, 2014.

Retrieved from: https://cds.cern.ch/record/1977865

Retrieved on: Mar. 20, 2018

16. P. Indelicato et al., "The Gbar project, or how does antimatter fall?," Hyperfine Interact., vol. 228, no. 1-3, pp. $141-150$, Oct. 2014 DOI: $10.1007 / \mathrm{s} 10751-014-1019-6$

17. H. S. Matis et al., "The BRAN luminosity detectors for the LHC," Nucl. Instrum. Methods Phys. Res. A., vol. 848, pp. 114 - 126, Mar. 2017. DOI: 10.1016/j.nima.2016.12.019

18. G. Gorine et al., "Ultra High Fluence Radiation Monitoring Technology for the Future Circular Collider at CERN," IEEE Trans. Nucl. Sci., 2018. DOI: 10.1109/TNS.2018.2797540

19. F. Warkusz, "The size effect and the temperature coefficient of resistance in thin films," J. Phys. D, vol. 11, no. 5, pp. 689 - 694, Apr. 1978. DOI: $10.1088 / 0022-3727 / 11 / 5 / 012$

20. N. Cabrera, N. F. Mott, "Theory of the oxidation of metals," Rep Prog Phys., vol. 12, pp. 163 - 184, 1949. DOI: 10.1088/0034-4885/12/1/308

21. M. O'Reilly et al., "Investigation of the oxidation behaviour of thin film and bulk copper," Appl. Surf. Sci., vol. 91, no. 1-4, pp. 152 - 156, Oct. 1995. DOI: 10.1016/0169-4332(95)oo111-5

22. S. K. Lee, H. C. Hsu, W. H. Tuan, "Oxidation Behavior of Copper at a Temperature below $300^{\circ} \mathrm{C}$ and the Methodology for Passivation," Mater. Res., vol. 19, no. 1, pp. $51-56$, Feb. 2016. DOI: 10.1590/1980-5373-MR-2015-0139

23. K. Väyrynen et al., "Low-Temperature Atomic Layer Deposition of Low-Resistivity Copper Thin Films Using $\mathrm{Cu}($ dmap)2 and Tertiary Butyl Hydrazine," Chem. Mater., vol. 29, no. 15, pp. 6502 - 6510, Jul. 2017. DOI: 10.1021/acs.chemmater.7bo2098 